\title{
Imperfections in perfect architecture: The orb web of spiders
}

\author{
Alain PASQUET ${ }^{1}$, Julia MARCHAL ${ }^{2}$, MylĖNe ANOTAUX ${ }^{1}$ and RAymond LEBORGNE ${ }^{1}$ \\ ${ }^{1}$ Université de Lorraine, Faculté des Sciences et Technologies, Laboratoire "Expression et Evolution des Comportements”, BP 239, \\ 54506 Vandoeuvre les Nancy - Cedex, France; e-mail: alain.pasquet@univ-lorraine.fr \\ ${ }^{2}$ UMR CNRS-MNHN 7179, Equipe "Mécanismes Adaptatifs et Evolution”, 1 Av. du Petit Château, 91800 Brunoy, France
}

Key words. Araneae, Zygiella x-notata, web construction, silky structures, anomalies, behavioural variability

\begin{abstract}
Among animal constructions, the orb webs of spiders are perfect geometrical architectural models. Webs are constructed by means of a succession of behaviours, which are often considered as instinctive and stereotyped. Anomalies in web structure are considered to be a consequence of variability in behaviour. In order to assess the variability in web structure, the spider Zygiella $x$-notata was allowed to build webs in the laboratory under standardized conditions. Webs $(\mathrm{n}=127)$ were examined for anomalies, which were defined as modifications in the geometrical structure. Ten anomalies in the structure of webs produced by females, aged from 16 to 21 days after their adult moult, are described. The frequency of anomalies per web varies from 5 to 98 . A very low percentage of the spiral elements of a web (less than 5\%) were affected. Anomalies in the radii were less numerous (mean $=3 \pm 3$ ) than those in the sticky spiral $($ mean $=21 \pm 11)$. Anomalies in the sticky spiral were different and more frequent in the peripheral than central parts of the webs. They were also relatively more numerous in the upper than in the lower part of the webs. Total number of anomalies was positively correlated with particular web characteristics (capture area, length of capture thread, width of the lower part of the web and mean distance between two spiral turns in the lower part) but not to the physical characteristics of the spiders (mass and size). Our results indicate that the orb webs of spiders, which are considered to be perfect geometrical structures, include anomalies mainly in the structure of the sticky spiral. These anomalies were the result of variability in behaviour during web building. In conclusion, their identification and description enable one to use them in spider webs as an index of behavioural variability.
\end{abstract}

\section{INTRODUCTION}

Animal constructions are the result of individual and/or collective activities (Hansell, 2005). All these constructions are the consequence of behaviour and require a certain level of cognitive complexity (Hansell \& Ruxton, 2008) or are self-organized (Stafford et al., 2011). In general, these constructions result from the use of environmental materials (Hymenoptera and bird nests) and more rarely the species produce the material used for the constructions. These constructions are the result of the interaction between genotype and environment and could be considered as extended phenotypes (Dawkins, 1982; Schaedelin \& Taborsky, 2009).

Of the 40,000 spider species, more than half build a trap, which consists of a silky structure that can intercept and retain prey (Foelix, 2011). Although there is a great diversity of these constructions, the geometrical webs are structurally regular. However, it is known that, in these webs, there are variations in the establishment of the attachment points, frame and radial threads, which involves modifications of the behavioural sequences such as thread addition and removal (Zschokke, 1996), but the sticky and the auxiliary spirals, which consist of a succession of lines of silk between two radii, are the result of repetitive behaviours.

The architecture of orb webs is always striking especially their geometrical regularity. The construction of such webs is described as follows: first, the spider fixes silk threads to supports, which constitute the frame in which the web is built. Second, the spider constructs the radii and then an auxiliary spiral, which is built from the centre outwards. Afterwards, the spider deposits a sticky spiral (capture thread) by moving from the periphery towards the centre of the web using the auxiliary spiral as a guide (Foelix, 2011). The geometry of the orb web is defined by the presence of radii around the hub and by the sticky spiral attached to these radii.

The design of the web is known to vary depending on environmental or internal conditions. Its structure may be affected by gravity (Witt et al., 1968, 1977; Vollrath \& Mohren, 1985), by drugs (Witt \& Reed, 1965; Reed \& Witt, 1968; Hesselberg \& Vollrath, 2004) or a morphological disability (missing or a shorter leg) (Vollrath, 1987; Pasquet et al., 2011). Moreover, web characteristics vary at the intraspecific and intra-individual levels (Herberstein \& Heiling, 1999; Heiling \& Herberstein, 2000) and are a direct reflection of spider behaviour (Zschokke \& Vollrath, 1995; Eberhard \& Hesselberg, 2012; Toscani et al., 2012).

In addition to the information on the variability in the structure of the orb webs, there are detailed descriptions of the building behaviour of the spiders; e.g. during the construction of the sticky spiral, the position of each thread depends on information perceived by the movements of the first pair of legs (L1) (Vollrath, 1987), and anomalies in web structure are associated with abnormal leg positions (Eberhard \& Hesselberg, 2012). There are several descriptions of stereotyped and repetitive behaviours, which can be translated into simple algorithms (Vollrath, 1992; Vollrath et al., 1997; Krink \& Vollrath, 
1997), but if spiders functioned like mathematical models, webs should have a perfect geometry and should vary in structure little at all and, any web defect may be ascribable to behavioural variation during construction (Quesada et al., 2011; Eberhard \& Hesselberg, 2012; Toscani et al., 2012).

Variations in the structures and geometry of the webs are used to quantify the influence of environmental factors such as space constraints (Ades, 1986; Krink \& Vollrath, 2000; Barrantes \& Eberhard, 2012; Hesselberg, 2012), parasite attacks (Eberhard, 2001, 2010; Gonzaga et al., 2010), or physiological factors such as stage of development (Eberhard, 2007, 2011; Hesselberg, 2010). There are other studies that take into account the regularity, irregularity or asymmetry, which result in the web being an imperfect orb (Witt et al., 1968; Ap Rhisart \& Vollrath, 1994; Vollrath et al., 1997; Hesselberg \& Vollrath, 2004; Coslovsky \& Zschokke, 2009; Nakata, 2010; Nakata \& Zschokke, 2010, Eberhard, 2011). In such cases, parameters are more linked to the general design of the web than to specific local anomalies, which may result from changes in behaviour during the construction of different parts of the web.

In conclusion, it is known that most of the anomalies in the structure of the web are due to changes in building behaviour. To identify, and describe the different anomalies in web structure, we allowed spiders to construct webs under standardized conditions in the laboratory in order to determine whether the variability in the number and types of anomalies was directly linked to the spider's behaviour. Such information can be used to quantify the variability in building behaviour under natural (pollutant, morphological disabilities, ageing) or experimental (drugs, space) conditions. So in the present paper, our aim is (i) to identify and describe anomalies in the radii and the sticky spiral of webs, (ii) to verify if there are relationships between the numbers of anomalies, characteristics of the webs, and morphological characteristics of the spiders.

\section{MATERIAL AND METHODS}

\section{Spider and laboratory conditions}

Zygiella x-notata (Clerck), Araneae, Araneidae, is an orbweaving spider abundant in the Paleartic region, which inhabits human constructions. In the north east of France, it reproduces at the end of summer or beginning of autumn and juveniles leave the egg sacs the following spring (Roberts, 1985). Immature females were collected from their natural environment. Adult moult was recorded in the laboratory by monitoring daily all the spiders and only young adult females were used. It is easy to breed this species in the laboratory under standardized conditions. In the laboratory the spiders were kept in plastic boxes $(10 \times 7 \times 2 \mathrm{~cm})$ (temperature $19^{\circ} \mathrm{C}$, light $12 \mathrm{~h}$ from 8 a.m. to 8 p.m.), fed with flies (Lucilia caesar) once per week, and supplemented with water. They did not build webs in the boxes.

For web building we used 16 to 21 days old females (mean age $=20$ days, $\mathrm{SE}=2$ days, $\mathrm{n}=127$ ). These spiders were placed in wooden frames $(50 \times 50 \times 10 \mathrm{~cm})$ enclosed between two panes of glass, which is a sufficient space for building a web as large as under natural conditions. The spinning of the webs was monitored daily. Only the first web built was used. A total of
127 webs constructed by 127 different spiders were used in the analysis (only 98 spiders were weighted; mean mass $=26.5 \mathrm{mg}$, $\mathrm{SE}=9.4 \mathrm{mg})$.

\section{Web characteristics}

The web of Zygiella $x$-notata is characterized by a free sector that lacks sticky threads and by a connecting thread between the centre of the web, and the retreat of the spider. The construction of this free sector is a result of the way this species constructs its sticky spiral. Zygiella x-notata reverses its direction of depositing the sticky thread instead of continuing in the same direction when it arrives at the last radius. Orb webs are two-dimensional objects that are easily quantifiable. We used the two parameters defined by Venner et al. (2001) for characterizing webs of Zygiella $x$-notata: the length of the thread in the sticky spiral (CTL), and the capture area (CA), which is the surface of the web minus the surface of the free sector and the central part of the web that lacks a sticky spiral. We recorded the total number of radii. We used also parameters characterizing the lower part of each web below the hub, which was, for this species, the most important part in terms of capturing prey (Nakata \& Zschokke, 2010). The parameters recorded were the width of the lower sector measured as the distance between the outer and innermost spiral turns, the number of spiral turns in this sector of the webs, and the mean distance between two successive spiral turns calculated by dividing the width of the lower part by the number (minus one) of spiral turns.

\section{Spider characteristics}

The mass of spiders was measured before it was placed into the frame (Sartorius, precision balance: $0.1 \mathrm{mg}$ ). Spider size was estimated by prosoma width. We recorded also the length of the first leg (L1) on the right side of each spider viewed from above. Leg length was obtained by adding the measurements for the four leg's segments (femur, patella-tibia, metatarsus and tarsus). This method was used because the measurements were done on dead spiders, for which legs could be never totally spread out. We measured the first leg because it is considered to have an important role in the construction and the characteristics of webs (Vollrath, 1987; Krink \& Vollrath, 1997). All size measurements were done using a dissecting stereoscope $(\times 100)$ (Wild) fitted with a micrometer. Twenty-one spiders were measured.

\section{Web anomalies}

Description of the anomalies

We defined anomalies as imperfections in a web that occurred during its construction. Anomalies could be in the radii or sticky spiral. The webs produced (about 30\%) during this study had few anomalies (Fig. 1), however, the aim of the study was the description of the anomalies in webs, such as those shown in Fig. 2.

\section{Anomalies in radii}

A radius was defined as a straight segment between the centre and a frame thread of a web (Fig. 2). According to this definition, we noted:

Supernumerary radius: It looks like a radius in the orb, which, however, does not originate from the centre of the web, and connects with sticky threads at each end.

Deviated radius: A radius that is not rectilinear but deviates by more than $5^{\circ}$ from a rectilinear trajectory starting from the centre of the web.

"Y" shape radius: A radius from the centre that splits into two separate segments that reach the frame threads at two different points. 


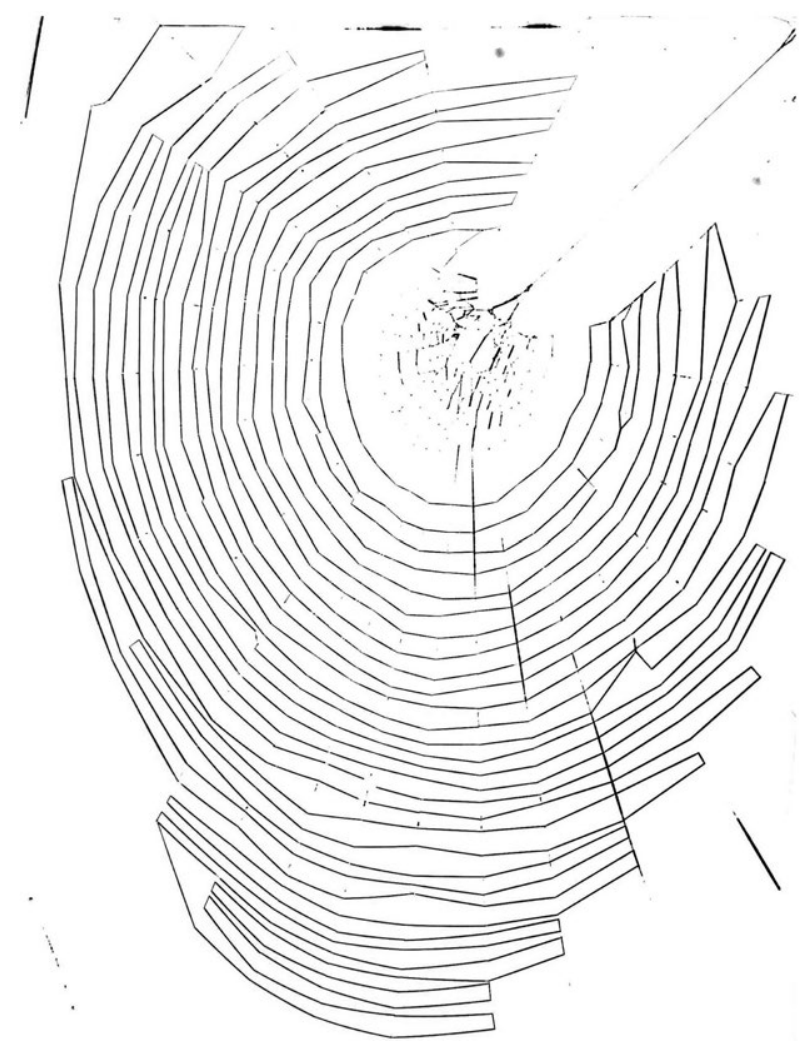

Fig. 1. An example of a web with few anomalies produced by a spider from the same population and reared in identical conditions to those that produce webs with anomalies.

\section{Anomalies in the sticky spiral}

Two successive radii define a sector in a web. In a sector, the spiral is made up of a series of sticky parallel threads (spiral units) attached to the radii. We noted seven anomalies that occurred in the structure in the sticky spiral.

Stop and return (Fig. 2): In a sector, two spiral units stop and end at one (triangular ending) or two points (rectangular ending) on a radius and are not on the same level as the spiral units on adjacent spiral turns. A stop and return occurs when the spiral units on either side of it, do not show stop and return on the same radius, which result in differences from the usual definition of returns (Zschokke, 2011).

Hole (Fig. 2): In a sector, a hole is defined if at least one spiral unit is absent and framed by at least two spiral units on each side.

Two spiral units stuck together (Fig. 2): Two consecutive spiral units are stuck together in a sector.

More than two spiral units stuck together (Fig. 2): Three or more consecutive spiral units are stuck together in a sector.

Discontinuity in the spiral threads (Fig. 2): In a sector other than the two adjacent sectors to the free sector, a spiral thread ends on a radius and is framed on either side by two threads that are not connected with it. It looks like spiral units stuck together but there is no contact with spiral units in adjacent sectors.

Non-parallel spiral units (Fig. 2): Two spiral units meet at a point on a radius ending triangular and prolonged into the next sector. This could be a special case of the anomaly "two spiral units stuck together".

Spiral units that zigzag (Fig. 2): A spiral goes in opposite direction in at least three consecutive sectors.

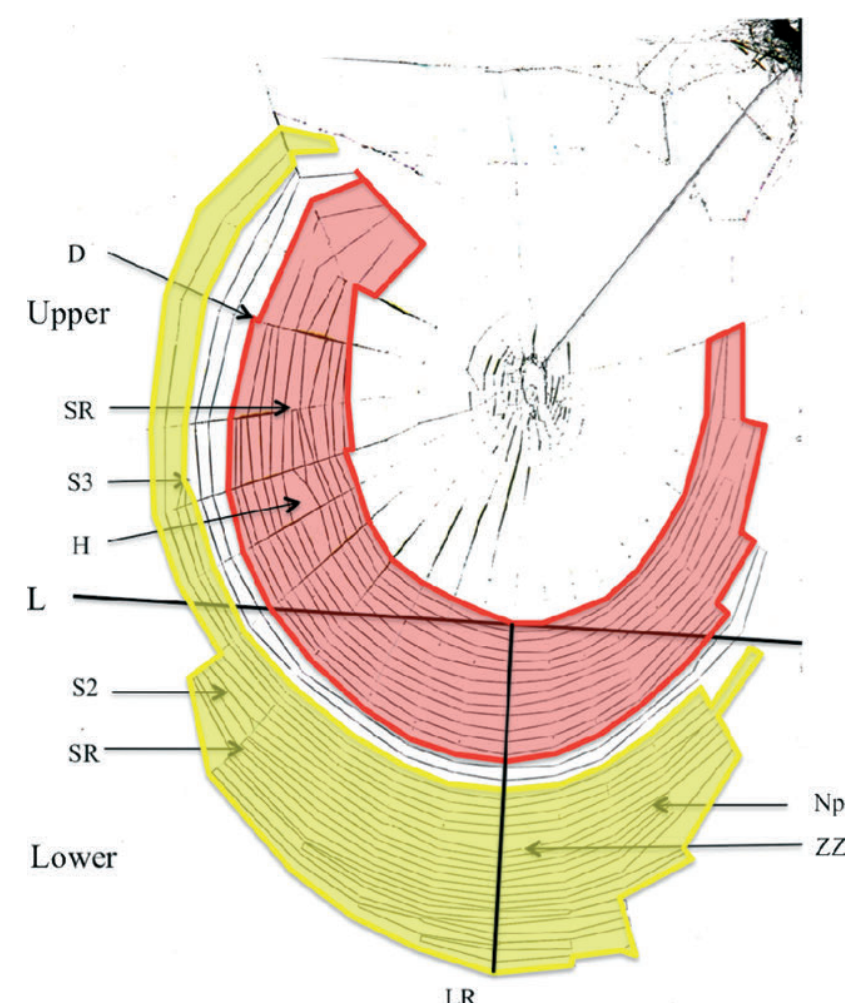

Fig. 2. Modalities of data sampling on a web of Zygiella. For the comparison of the upper and lower parts, L divides each web into two parts. This line (L) is perpendicular to the longest radius in the lower part of the web and the line (LR) is tangential to the innermost spiral turn. To compare the number of anomalies in the inner and outer-most spiral turns, each web was divided into two parts: an inner (red colour) and outer part (yellow colour). The anomalies counted in each corresponding area were: D - discontinuities in the spiral threads; $\mathrm{H}$ - holes; $\mathrm{Np}$ - non-parallel spiral units; SR - stop and return; S2 - two threads stuck together; S3 - more than two threads stuck together; $\mathrm{ZZ} \mathrm{-} \mathrm{spiral} \mathrm{units} \mathrm{that} \mathrm{zigzag.}$

\section{Data analysis}

For each web, we counted all the anomalies and presented the average number per web for 127 webs. The anomalies were placed in one of two groups: anomalies associated with the radii, which are constructed during the second stage of web construction, and those associated with the sticky spiral, which is constructed during the fourth and final stage of web building (Foelix, 2011).

Then the distribution of the anomalies in the sticky spiral of a sample of 40 webs chosen at random from among the 127 webs was analyzed. This was done because to take into account all the webs $(\mathrm{n}=127)$ would have been very time-consuming and unlikely to give more statistical information. We counted all the spiral units and anomalies on each web. To determine the number of spiral units, we followed the path used by the spider to lay the sticky spiral using photographs of each web. We compared the number of anomalies in the lower and upper parts of the webs (Fig. 2): by dividing the webs in two parts based on a line (L) perpendicular to the longest radius (LR) in the lower part of the web that runs tangential to the innermost spiral turn. We counted the number of spiral units and the number of anomalies in each part (Fig. 2). As the numbers of spiral units were not the same in the upper and lower parts of webs, we expressed the number of anomalies as a percentage of the spiral 
units of each part $(\%$ anomalies $=$ number of anomalies $/$ number of spiral units $\times 100$ ). We compared also, the number of anomalies in the outermost and innermost spiral turns of the whole orb (Fig. 2). To do that, the number of anomalies in two hundred spiral units of the outer thread and two hundred of the inner thread of the spiral (near the centre of the web) were counted When a web was small and had less than four hundred spiral units in the sticky spiral, we counted the total number of spiral units and divided it by two. These small webs were included in the analysis, because they made more than $20 \%$ of the total number of webs studied and were present in the set of webs chosen at random.

\section{Statistical analysis}

For comparing the number of anomalies in the lower and upper parts of the web, the number of anomalies per web was expressed as the number per $\mathrm{cm}$ of sticky spiral thread (CTL). We tested for correlations between the number of anomalies per web, other web parameters and spider physical characteristics using a simple parametric linear regression. We tested for allometric relationship between the number of anomalies per web and web size [length of the sticky spiral (CTL) and capture area (CA)] and as an allometric relationship is characterized by a power equation, we looked for a linear correlation between the logarithmic transformation of the number of anomalies and that of CTL or CA. For the analyses of the distribution of anomalies on the sticky spiral we used a two-tailed paired $t$ test.

Tests were done using Statview software 5.0 on a MacIntosh platform. The results were expressed as means and Standard Errors (SE), and the statistical significance was considered for $\mathrm{p}$ $<0.05$.

\section{RESULTS}

\section{Anomalies}

A total of 127 webs were analyzed (Table 1). The total number of anomalies per web varied from 5 to 98 (mean $=35 \pm 1, \mathrm{n}=127$ ) (Fig. 3). Except for the supernumerary radii that were recorded in only $15 \%$ of the webs, the majority of the anomalies (6/10) occurred in more than $80 \%$ of the webs (deviating radii, stop and return, holes, two spiral units stuck together, discontinuities in spiral units and spiral units that zigzag, Table 1).

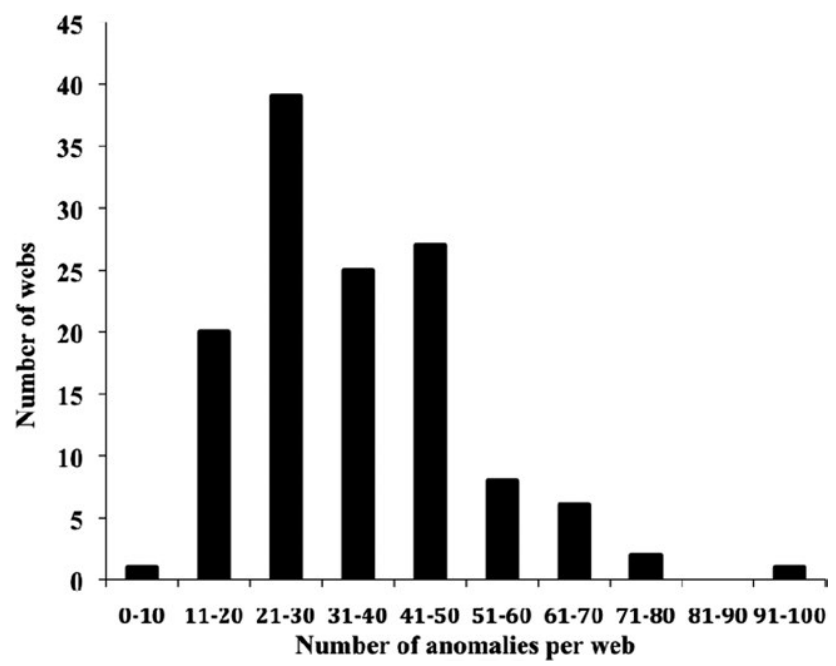

Fig. 3. Histogram of the number of webs with different total number of anomalies.

Anomalies in radii were less numerous than in the sticky spiral (mean per web for radii $=3.1, \mathrm{SE}=2.7$, mean per web for the sticky spiral $=21.4, \mathrm{SE}=11.4, \mathrm{n}=$ 127). Stop and return was the most frequent of the anomalies on the sticky spiral (mean per web $=10.0, \mathrm{SE}$ $=4.8, \mathrm{n}=127$ ). There was a positive relationship between the numbers of anomalies in the radii and the sticky spiral $\left(\mathrm{R}^{2}=0.18, \mathrm{n}=127, \mathrm{~F}=29.3, \mathrm{p}<0.0001\right)$.

The number of anomalies relatively to that of spiral units per web is very low (number of spiral units: $\mathrm{m}=$ $486, \mathrm{SE}=28$, number of anomalies: $\mathrm{m}=16, \mathrm{SE}=1$, percentage of anomalies: $\mathrm{m}=3.6 \%, \mathrm{SE}=0.2 \%, \mathrm{n}=40$ ). Anomalies on the sticky spiral were not equally distributed, as there were relatively more in the upper than in the lower part of webs (upper part: $\mathrm{m}=4.8 \%, \mathrm{SE}=0.4 \%$, lower part: $\mathrm{m}=3.0 \%, \mathrm{SE}=0.3 \%, \mathrm{t}=4.80, \mathrm{df}=39, \mathrm{p}<$ $0.001)$. Stop and return, non-parallel spiral units and discontinuities in the spiral thread were relatively more numerous in the upper part of webs (stop and return: $\mathrm{t}=$ 2.04 , df $=39, \mathrm{p}=0.048$, non-parallel spiral units: $\mathrm{t}=$

TABLE 1. Number of different anomalies recorded in the webs of Zygiella x-notata webs. The numbers of anomalies were recorded in 127 webs spun under laboratory conditions and used to calculate their relative percentages (percentage of webs with a given type of anomaly and mean number of anomalies per web).

\begin{tabular}{lcccc}
\hline Anomalies & $\begin{array}{c}\text { Total number } \\
\text { of anomalies }\end{array}$ & $\begin{array}{c}\text { Percentage of } \\
\text { each anomaly }\end{array}$ & $\begin{array}{c}\text { Percentage of webs in which } \\
\text { an anomaly was present }\end{array}$ & $\begin{array}{c}\text { Mean number of anomalies } \\
\text { per web (SE) }\end{array}$ \\
\hline Deviated radii & 306 & $6.4 \%$ & $84 \%$ & $2,6(2,0) \mathrm{n}=107$ \\
Y shaped radii & 102 & $2.1 \%$ & $45 \%$ & $1,6(0,1) \mathrm{n}=57$ \\
Supernumerary radii & 21 & $0.4 \%$ & $15 \%$ & $1,1(0,3) \mathrm{n}=19$ \\
Returns & 1402 & $29.3 \%$ & $100 \%$ & $10,0(4,8) \mathrm{n}=127$ \\
Hole & 778 & $16.2 \%$ & $100 \%$ & $5,6(2,5) \mathrm{n}=127$ \\
Two spiral units stuck together & 781 & $16.3 \%$ & $98 \%$ & $5,8(5,3) \mathrm{n}=124$ \\
More than two spiral units stuck together & 138 & $2.9 \%$ & $46 \%$ & $2,2(2,6) \mathrm{n}=58$ \\
Discontinuity & 726 & $15.2 \%$ & $93 \%$ & $6,1(4,0) \mathrm{n}=118$ \\
Non parallel spiral units & 99 & $2.1 \%$ & $51 \%$ & $1,4(0,7) \mathrm{n}=65$ \\
Spiral thread that zigzag & 437 & $9.1 \%$ & $97 \%$ & $3,3(1,4) \mathrm{n}=123$ \\
\hline Total & 4790 & $100 \%$ & & \\
\hline
\end{tabular}


2.05, $\mathrm{df}=39, \mathrm{p}=0.047$ and discontinuities: $\mathrm{t}=5.05, \mathrm{df}=$ $39, \mathrm{p}<0.001)$. There was no difference in the distribution of the other anomalies. The comparison of the outer and innermost parts of webs revealed more anomalies in the outer than in the innermost part of the spiral ( $\mathrm{t}$-test, $\mathrm{t}=$ 5.02, $\mathrm{df}=39, \mathrm{p}<0.001)$. Stop and return, holes and two spiral units stuck together were the most numerous anomalies and were more frequent in the outer part of the sticky spiral (stop and return: t-test, $\mathrm{t}=8.04$, df $=39, \mathrm{p}<$ 0.0001 , holes: t-test, $\mathrm{t}=3.99, \mathrm{df}=39, \mathrm{p}<0.001$ and two spiral units stuck together: $\mathrm{t}$-test, $\mathrm{t}=4.15$, df $=39, \mathrm{p}<$ $0.001)$ whereas there was no difference in the distribution of the other anomalies.

\section{Relationships between the number of anomalies and web parameters}

We tested for the existence of an allometric relationship between the logarithm of the number of anomalies and that of the size of the webs using a linear correlation. The number of anomalies per web increased with increase in the size of the CA $\left(\mathrm{R}^{2}=0.07, \mathrm{n}=127, \mathrm{~F}=10.2, \mathrm{p}=\right.$ 0.002). Also, the longer the sticky spiral (CTL) the greater was the number of anomalies $\left(\mathrm{R}^{2}=0.14, \mathrm{n}=127\right.$, $\mathrm{F}=20.1, \mathrm{p}<0.0001)$. Thus, the number of anomalies was linked to web size by a power function and increased more rapidly than the size of the web (CA) or the length of the silk thread in the sticky spiral (CTL).

The total number of anomalies was positively correlated with the length and the number of spiral turns in the lower part of webs (length in the lower part of webs: $\mathrm{R}^{2}=$ $0.07, \mathrm{n}=127, \mathrm{~F}=6.4, \mathrm{p}=0.013$; number of spiral turn in lower part of webs: $\mathrm{R}^{2}=0.15, \mathrm{n}=127, \mathrm{~F}=22.4, \mathrm{p}<$ $0.001)$. On the other hand, there was no relation between the number of anomalies and the number of radii $\left(\mathrm{R}^{2}=\right.$ $0.02, \mathrm{n}=127, \mathrm{~F}=2.55$, N.S.) or with the mean distance between spiral turns in the lower part of webs $\left(\mathrm{R}^{2}=0.00\right.$, $\mathrm{n}=127, \mathrm{~F}=0.06$, N.S.).

\section{Relationships between the number of anomalies and spider characteristics}

There was no relation between the total number of anomalies and the mass of the spiders (total number of anomalies: $\mathrm{R}^{2}=0.01, \mathrm{n}=98, \mathrm{~F}=2.1$, N.S.). In addition there was no correlation between the number of anomalies and the parameters used to measure spider size (length of first leg: $\mathrm{R}^{2}=0.11, \mathrm{n}=21, \mathrm{~F}=2.0$, N.S., and width of prosoma: $\mathrm{R}^{2}=0.13, \mathrm{n}=21, \mathrm{~F}=2.6$, N.S.).

\section{Relationships between types of anomalies, web parameters and spider characteristics}

Simple linear regression between numbers of anomalies, web parameters and spider characteristics revealed no significant relations except for stop and return, which was correlated positively with all web characteristics measured $\left(\mathrm{CA}: \mathrm{R}^{2}=0.18, \mathrm{n}=127, \mathrm{~F}=29.4, \mathrm{p}\right.$ $<0.0001$, CTL: $\mathrm{R}^{2}=0.40, \mathrm{n}=127, \mathrm{~F}=83.1, \mathrm{p}<0.0001$, width of the lower part of webs: $\mathrm{R}^{2}=0.25, \mathrm{n}=127, \mathrm{~F}=$ 43.2, $\mathrm{p}<0.0001$, number of spiral turns in the lower part of webs: $\mathrm{R}^{2}=0.58, \mathrm{n}=127, \mathrm{~F}=180.4, \mathrm{p}<0.0001$, and number of radii: $\left.\mathrm{R}^{2}=0.10, \mathrm{n}=127, \mathrm{~F}=13.9, \mathrm{p}<0.001\right)$. The number of stop and return was also positively corre- lated with spider mass $\left(\mathrm{R}^{2}=0.04, \mathrm{n}=98, \mathrm{~F}=2.2, \mathrm{p}=\right.$ $0.03)$.

\section{DISCUSSION}

Our study reveals that there are anomalies in the structure of the orb webs of Zygiella x-notata. All the spiders studied built webs with the global architecture of an orb-web and local imperfections in the radii and sticky spiral. As the spiders built their web in standardized frames and as they were checked just after the construction was completed, the anomalies could only be due to variations in behaviour during web building. The building of orb webs involves repetitive behaviours, which can be simulated by algorithms (Krink \& Vollrath, 1997). The anomalies in the sticky spiral described here are local and involve very few successive spiral units, and are possibly the consequence of "alterations" in the execution of repetitive behaviours (steps in the algorithms). The total number of anomalies in the sticky spiral varied from web to web with a low frequency of anomalies relatively to the number of spiral units. So, these anomalies could be the consequence of individual temporary variations in behaviours during web building.

All the anomalies were more numerous in the large webs. The increase is not directly proportional to web size as the number of anomalies increases more rapidly than the size of the webs. The number of anomalies in the sticky spiral is directly linked to the number of silk units that make up the final sticky spiral. It is not related to the physical characteristics of the spiders (mass and size), but to web characteristics (size of the capture area, length of silky thread deposited, inter-spiral distance). It is known that the web characteristics vary depending on the space available (Ades, 1986; Krink \& Vollrath, 2000; Barrantes \& Eberhard, 2012; Hesselberg, 2012) previous prey capture (Lubin \& Henschel, 1992; Henshel \& Lubin, 1996; Pasquet et al., 1999), prey size (Thevenard et al., 2004; Blackledge \& Zevenbergen, 2006; Abrenicot-Adamat et al., 2009), presence of conspecifics (Leborgne \& Pasquet, 1986), internal state of the spider (Venner et al., 2003) and experience (Heiling \& Herberstein, 1999, 2000; Venner et al., 2000; Sensenig et al., 2010), or stage of development of the spider (Eberhard, 2007, 2011; Hesselberg, 2010). As the number of anomalies is linked to web characteristics, it might be affected by these different factors, but under controlled laboratory conditions, the influence of these environmental factors was reduced or negligible. Therefore, in this case, the number of anomalies is considered to be a characteristic of the spiders and independent of environmental factors.

The number of anomalies recorded in Zygiella webs was very low (less than $5 \%$ of the total sticky spiral units were affected). Under controlled conditions, our spiders made very few behavioural errors during web construction. This is a notable phenomenon, and raises the question: are these errors (leading to web anomalies) randomly distributed or more likely to occur in particular parts of webs, as it is known that the different parts of web involve different technics of construction. 
The comparisons of the spiral units built at the beginning and end of the construction of webs revealed differences in types and number of anomalies (i.e. more threads stuck together and stop and return at the beginning than at the end). This does not indicate a shortage of silk. It is known that the amount of silk can be a limiting factor in web construction (Eberhard, 1988) and may affect the number of anomalies recorded at the beginning and end of web building. It does not support a tiredness hypothesis, as there are more errors at the beginning of the construction than at the end. Thus, these differences could be correlated with changes in web building behaviours.

As in many other orb web spiders, during the construction of the sticky spiral, Zygiella x-notata used the length of its forelegs (L1) to evaluate the distance between two radii. The building of the sticky spiral begins with the construction of the outermost part of the spiral and in this case the distance between two radii is larger than the length of the forelegs, so the spider has to move to the centre of the web to find the next radius (Vollrath, 1987; Eberhard, 2011; Foelix, 2011). At the end of the construction of the sticky spiral when the spider is near the centre, the distance between two radii is small and the spider can locate directly the next radius with its forelegs. That is, between the beginning and end of the construction of the sticky spiral, spiders modify their silk laying behaviour and these changes in behaviour in spiral unit construction may differentially affect the probability of occurrence of anomalies.

The comparison of the incidence of anomalies in the upper and lower parts of webs also revealed that they are not equally distributed, as there are relatively more stop and return, non-parallel threads and discontinuities in the upper than lower parts of webs. This could indicate that anomalies occur during the different sequences of web building. When building its web, a spider has to change frequently the orientation of the axis of its body relative to the position (vertical or horizontal) of the deposited thread. Even if we had not observed the building behaviour of spiders during the construction of the different parts of webs, we know that during the laying of the spiral units, some behavioural errors may occur (Toscani et al., 2012). By studying the building behaviour and recording the incidence of anomalies, such as two threads stuck together and non-parallel threads, this author found that these two behavioural errors were directly linked to the position of the fourth leg and of the abdomen. As the positions of the body of the spider were not the same when the spider deposited the spiral units in the different parts of webs, this could be the origin of behavioural errors that result in the anomalies.

Moreover, it is known that behavioural decisions may be influenced by both the position of the temporary nonsticky and sticky spirals, which involves short-term memory (Eberhard \& Hesselberg, 2012) and could affect the final silky structure. In conclusion, some of the anomalies in orb webs observed in this study were probably the result of behavioural errors based on a poor use of local information (distance to the temporary spiral or to a previous junction with a radius).

Thus, anomalies in orb webs could be a good model for studying variations in behaviour induced either by environmental factors (changes in prey availability, presence of conspecifics, space) or internal factors (development, age, physiological state). For this we have to consider anomalies at two different levels, those anomalies (imperfections in the geometrical structure) that could be the result of behavioural decisions and those that could be the result of behavioural errors during web construction. In the first group, could be supernumerary radii or $\mathrm{Y}$ shaped radii, or stop and return. These anomalies could be seen as the result of voluntary acts of a spider carried out in order to increase the area of its web and could be used as index of the foraging behaviour of the spider. On the other hand, anomalies resulting from behavioural errors (i.e. Toscani et al., 2012) such as holes, discontinuities in the spiral threads, two or more spiral units stuck together, may indicate some neural dysfunction. For example, different studies showed that several substances (i.e. drugs, and pharmacological products) affect web building behaviour and might also affect the design of orb-webs (Witt et al., 1968; Hesselberg \& Vollrath, 2004). If alteration in web construction behaviour affects the typical geometry of an orb web (Reed et al., 1970), then the anomalies we observed in Zygiella webs could be similar to those attributed to the effect of drugs or pharmacological products (Hesselberg \& Vollrath, 2004) or age (Anotaux et al., 2012). We agree with these authors that the different substances or age affect behaviour through their action on the brain then the locomotory activity or coordination of spiders during web construction could be affected.

Thus, taking all these facts into consideration it is concluded that anomalies recorded in the web structure of orb-web spiders are likely to be the consequences of individual variations in behaviour. That is under controlled laboratory conditions in which the spiders did not suffer environmental constraints, and the variability in web design did not appear to be linked to web characteristics or a spider's state but more to errors in thread laying. These results suggest that studies of the anomalies in the sticky spiral could be a useful way to investigate the influence of CNS on the carrying out of a task that involves repetitive behaviours.

ACKNOWLEDGEMENTS. This work was done at the UHP University Nancy, funded by a grant from the CNRS (interdisciplinary program PIR, “Ageing and Longevity"). We thank L. Bahans for her help with rearing spiders and Y. Lubin and A.F.G. Dixon for correcting the English version of this manuscript.

\section{REFERENCES}

Abrenicot-Adamat L.R., Torres M.A., Barrion A.A., Dupo A.L. \& Demayo C.G. 2009: The influence of prey on size, capture area, and mesh height of the orb-web of the garden spider Argiope aemula (Walckenaer 1841) (Araneae; Araneidae). - Egypt. Acad. J. Biol. Sci. 1: 65-71. 
ADEs C. 1986: A construcao da teia geometrica como programa comportemental. - Cienca Cult. 38: 760-775.

Anotaux M., Marchal J., Châline N., Desquilbet L., Leborgne R., Gilbert C. \& Pasquet A. 2012: Ageing alters spider orb-web construction. - Anim. Behav. 84: 1113-1121.

Ap Rhisiart A. \& Vollrath F. 1994: Design features of a spider orb web. - Behav. Ecol. Sociobiol. 5: 280-287.

BARRANTES G. \& Eberhard W.G. 2012: Extreme behavioural adjustments by an orb-web spider to restricted spaces. Ethology 118: 438-449.

Blackledge T.A. \& Zevenbergen J.M. 2006: Mesh width influences prey retention in spider orb-webs. - Ethology 112: 1194-1201.

Coslovsky M. \& ZschokKe S. 2009: Asymmetry in orb-webs: an adaptation to web building costs. - J. Insect Behav. 22: 29-38.

DawkINS R. 1982: The Extended Phenotype. Oxford University Press, Oxford, UK, 320 pp.

EBERHARD W.G. 1988: Behavioral flexibility in orb web constructions: effects of supplies in different silk glands and spider size and weight. — J. Arachnol. 16: 295-302.

EBERHARD W.G. 2001: Under the influence: webs and building behavior of Plesiometa argyra (Araneae, Tetragnathidae) when parasitized by Hymenoepimecis argyraphaga (Hymenoptera, Ichneumonidae). — J. Arachnol. 29: 354-366.

EBERHARD W.G. 2007: Miniaturized orb-weaving spiders: behavioural precision is not limited by small size. - Proc. $R$. Soc. (B) 274: 2203-2209.

EBERHARD W.G. 2010: Recovery of spiders from the effects of parasitic wasps: implications for fine-tuned mechanisms of manipulation. - Anim. Behav. 79: 375-383.

EBERHARD W.G. 2011: Are smaller animals behavioural limited? Lack of clear constraints in miniature spiders. - Anim. Behav. 81: 813-823.

Eberhard W.G. \& Hesselberg T. 2012: Cues that spiders (Araneae: Araneidae, Tetragnathidae) use to build orbs: lapses in attention to one set of cues because of dissonance with others? - Ethology 118: 610-620.

Foelix R.F. 2011: Biology of Spiders. 3rd ed. Oxford University Press, Oxford, $411 \mathrm{pp}$

Gonzaga M.O., Sobczak J.F., Vasconcellos-Neto J., Loffredo A.P.S. \& Penteado-Dias A.M. 2010: The effect of host body size and web aggregation on parasitism of Nephila clavipes (Araneae, Nephilidae) by Hymenoepimecis bicolor (Hymenoptera, Ichneumonidae). - Ethol. Ecol. Evol. 22: 151-165.

Hansell M. 2005: Animal Architecture. Oxford University Press, Oxford, $334 \mathrm{pp}$

Hansell M. \& Ruxton G.D. 2008: Setting tool use within the context of animal construction. - Trends Ecol. Evol. 23: $73-78$.

Heiling A.M. \& Herberstein M.E. 1999: The role of experience in web building spiders (Araneidae). - Anim. Cognit. 2: 171-177.

HeILING A.M. \& Herberstein M.E. 2000: Interpretations of orbweaving variability: a review of past and current ideas. Ekológia 19: 97-106.

HeNsCHEL J.R. \& LuBIN Y. 1996: Environmental factors affecting the web and activity of a psammophidus spider in the namib desert. - J. Arid. Environ. 22: 173-189.

Herberstein M.E. \& Heiling A.M. 1999: Asymmetry in spider orb web (Araneidae): a result of physical constraints? Anim. Behav. 58: 1241-1246.

Hesselberg T. 2010: Ontogenetic changes in web design in two orb-web spiders. — Ethology 116: 535-545.
Hesselberg T. 2012: Web-building flexibility differs in two spatially constrained orb spiders. - J. Insect Behav. 26: 283-303.

Hesselberg T. \& Vollrath F. 2004: The effects of neurotoxins on web-geometry and web-building behaviour in Araneus diadematus $\mathrm{Cl}$. - Physiol. Behav. 82: 519-529.

Krink T. \& Vollrath F. 1997: Analysing spider web-building behaviour with rule-based simulations and genetic algorithms. - J. Theor. Biol. 185: 321-331.

Krink T. \& Vollrath F. 2000: Optimal area use in orb-webs of the spider A. diadematus. - Naturwissenschaften 87: 90-93.

LEBORgNe R. \& PASQuet A. 1986: Influence of aggregative behaviour on space occupation in the spider Zygiella x-notata (Clerck). - Behav. Ecol. Sociobiol. 20: 203-208.

LuBIN Y. \& Henschel J.R. 1992: The influence of food supply on foraging behaviour in a desert spider. - Oecologia 105: 64-73.

NAKATA K. 2010: Does ontogenetic change in orb-web asymmetry reflect biogenetic law? - Naturwissenschaften 97: 1029-1032.

Nakata K. \& ZschokKe S. 2010: Upside-down spider build upside-down orb webs: web asymmetry, spider orientation and running speed in Cyclosa. - Proc. Biol. Sci. 277: 3019-3025.

Pasquet A., Lubin Y. \& Leborgne R. 1999: Previous foraging success influences web building in $S$. lineatus (Eresidae). Behav. Ecol. 10: 115-121.

Pasquet A., Anotaux M. \& Leborgne R. 2011: Loss of legs: is it or not a handicap for an orb-weaving spider? - Naturwissenschaften 98: 557-564.

Quesada R., Triana E., Vargas G., Seid M., Douglass J., EberHARD W.G. \& WCISLO W.T. 2011: The allometry of CNS size and consequences of miniaturization in orb-weaving spiders. - Arth. Struct. Devel. 40: 521-529.

ReEd C.F. \& WitT P.N. 1968: Progressive disturbance of spider web geometry caused by two sedative drugs. - Physiol. Behav. 3: 119-124.

Reed C.F., Witt P.N., Scaboro M.B. \& Peakall D.B. 1970: Experience and the orb web. - Devel. Psychobiol. 3: 251-265.

RoBerts M.J. 1985: Collins Field Guide: Spiders of Britain and Northern Europe. Delachaux and Niestlé, Paris, 384 pp.

SchaEdelin F.C. \& TABORSKY M. 2009: Extended phenotypes as signals. - Biol. Rev. 84: 293-313.

Sensenig A., Agnarsson I., Gondek T. \& Blackledge T.A. 2010: Webs in vitro and in vivo: spiders alter their orb-web spinning behavior in the laboratory. - J. Arachnol. 38: 183-191.

Stafford R., Williams G.A. \& Davies M.S. 2011: Robustness of self-organised systems to changes in behaviour: an example from real and simulated self-organised snail aggregations. - PLOS ONE 6: e22743.

Thevenard L., Leborgne R. \& Pasquet A. 2004: Web building management in an orb-weaving spider Zygiella x-notata: influence of prey and conspecifics. - C. R. Biol. 327: 84-92.

Toscani C., Leborgne R. \& Pasquet A. 2012: Analysis of web building anomalies in the orb weaving spider Zygiella x-notata (Araneae, Araneidae). Arachnol. Mitt. 43: 79-83.

Venner S., Pasquet A. \& Leborgne R. 2000: Web-building behaviour in the orb weaving spider Zygiella $x$-notata: influence of experience. - Anim. Behav. 59: 603-611.

Venner S., Thevenard L., Pasquet A. \& Leborgne R. 2001: Estimation of the web's capture thread length in the orbweaving spiders: determination of the most efficient formula. - Ann. Entomol. Soc. Am. 94: 490-496. 
Venner S., Bel-Venner M.C., Pasquet A. \& Leborgne R. 2003 : Body-mass dependent cost of web-building in an orb-weaving spider Zygiella x-notata. - Naturwissenschaften $\mathbf{9 0}$ 269-272.

VollRath F. 1987: Altered geometry of webs in spiders with regenerated leg. - Nature 328: 247-248.

Vollrath F. 1992: Analysis and interpretation of orb spider exploration and web-building behavior. - Adv. Study Behav. 21: 147-199.

Vollrath F. \& Moren W. 1985: Spiral geometry of the garden spider's orb web. — Naturwissenschaften 72: 666-668.

Vollrath F., Downes M. \& KraKow S. 1997: Design variability in web-geometry of an orb-weaving spider. — Physiol. Behav. 62: 735-743.

Witt P.N., Peakall D.B. \& Gause R.L. 1977: Spider web building in outer space; evaluation of records from the Skylab spider experiment. — J. Arachnol. 4: 115-124.
Witt P.N. \& Reed C.F. 1965: Spider-web building. Measurement of web geometry identifies in a complex invertebrate behavior pattern. - Science 149: 1190-1197.

Witt P.N., Reed C.F. \& Peakall D.B. 1968: A Spider's Web: Problems in Regulatory Biology. Springer, New York, 107 pp.

ZschокKE S. 1996: Early stages of web construction in Araneus diadematus Clerck. — Rev. Suisse Zool. 2: 709-720.

ZschoкKe S. 2011: Spiral and web asymmetry in the orb web of Araneus diadematus (Araneae, Araneidae). - J. Arachnol. 39: 358-362.

ZsCHOKKE S. \& VollRAth F. 1995: Web construction patterns in a range of orb-weaving spiders (Araneae). - Eur. J. Entomol. 92: 523-541.

Received May 10, 2012; revised and accepted March 7, 2013 